\title{
Zinc Influx Restricts Enterovirus D68 Replication
}

\author{
Shunan Liu', Xia Cao ${ }^{1 *}$, Haoran $\mathrm{Guo}^{2}$ and Wei Wei ${ }^{2 *}$ \\ ${ }^{1}$ Department of Pharmacology, School of Pharmacy, Jilin University, Changchun, China, ${ }^{2}$ Key Laboratory of Organ \\ Regeneration and Transplantation of the Ministry of Education, Institute of Translational Medicine, Institute of Virology and \\ AIDS Research, The First Hospital of Jilin University, Changchun, China
}

OPEN ACCESS

Edited by: Xin Yin,

Harbin Veterinary Research Institute Chinese Academy of Agricultural Sciences (CAAS), China

Reviewed by: Weihua Huang, New York Medical College, United States Deyan Chen, Nanjing University Medical School, China

*Correspondence: Wei Wei wwei6@jlu.edu.cn Xia Cao caoxia@jlu.edu.cn

Specialty section: This article was submitted to Virology,

a section of the journal Frontiers in Microbiology

Received: 30 July 2021 Accepted: 16 September 2021 Published: 13 October 2021

Citation:

Liu S, Cao X, Guo H and Wei W (2021) Zinc Influx Restricts Enterovirus D68 Replication.

Front. Microbiol. 12:748546. doi: 10.3389/fmicb.2021.748546
Enterovirus D68 (EV-D68) is a respiratory viral pathogen that causes severe respiratory diseases and neurologic manifestations. Since the 2014 outbreak, EV-D68 has been reported to cause severe complications worldwide. However, there are currently no approved antiviral agents or vaccines for EV-D68. In this study, we found that zinc ions exerted substantial antiviral activity against EV-D68 infection in vitro. Zinc salt treatment potently suppressed EV-D68 RNA replication, protein synthesis, and infectious virion production and inhibited cytopathic effects without producing significant cytotoxicity at virucidal concentrations $\left(\mathrm{EC}_{50}=0.033 \mathrm{mM}\right)$. Zinc chloride $\left(\mathrm{ZnCl}_{2}\right)$ treatment moderately inhibited EV-D68 attachment. Time-dose analysis of EV-D68 structural protein VP1 synthesis showed stronger suppression of VP1 in the culture medium than that in the cell lysates. Furthermore, a zinc ionophore, pyrrolidine dithiocarbamate, which can transport zinc ions into cells, also enhanced the anti-EV-D68 activity of $\mathrm{ZnCl}_{2}$ treatment. Taken together, our results demonstrated that the enhancement of zinc influx could serve as a powerful strategy for the therapeutic treatment of EV-D68 infections.

Keywords: enterovirus, EV-D68, zinc influx, pyrrolidine dithiocarbamate, antiviral agents

\section{INTRODUCTION}

Enterovirus D68 (EV-D68) of the Enterovirus genus (Picornaviridae family) was first isolated in 1962 (Schieble et al., 1967; D'Esposito and Postle, 2015). It has been found that EV-D68 particularly affects children, occasionally causing respiratory illness (Nafisa et al., 2021). It was initially considered a rare virus until the first large outbreak in 2014 (Midgley et al., 2014). Importantly, recent studies have found that EV-D68 infection not only triggers sporadic respiratory illness but is also associated with serious neurological conditions as acute flaccid myelitis (AFM), mainly occurs in pediatric patients (Midgley et al., 2015; Chang et al., 2017). Although EV-D68 is considered to be the main causative agent of AFM, therapeutic drug or treatment specifically for EV-D68 infections is still not available globally.

Enterovirus D68 is a non-enveloped, single-stranded, positive-sense RNA virus. Unlike most enteroviruses that encode ORF2p/uORF (Guo et al., 2019; Lulla et al., 2019), the EV-D68 genome has only one open reading frame (ORF). The ORF encodes a polyprotein precursor, which can be processed by viral proteases into four structural proteins (VP1, VP2, VP3, and VP4) and seven nonstructural proteins (2A, 2B, 2C, 3A, 3B, 3C, and 3D). The known EVD68 receptors are sialic acid and ICAM-5 (Liu et al., 2015a; Baggen et al., 2016; Wei et al., 2016). Thus far, the pathogenesis of EV-D68 infections remains poorly understood. 
Zinc ion is the second most abundant transition metal ion in human bodies. It plays vital roles in many biological processes, including cell fate and development, gene transcription, and viral infection. Zinc ion inhibits viral replication by impairing polyprotein processing (Lanke et al., 2007), inhibiting transcription (Haraguchi et al., 1999; Fenstermacher and DeStefano, 2011), interfering with viral-cell membrane fusion to block viral binding and attachment (Suara and Crowe, 2004), and inhibiting viral genome replication (Korant et al., 1974; Bracha and Schlesinger, 1976). Furthermore, zinc plays a crucial role in enhancing host resistance to viral infections by sustaining immune homeostasis. Conversely, zinc deficiency impairs the cellular immune response and weakens cellular immunity. Compounds that are capable of transporting zinc into cells or redistributing cellular zinc exert a viral inhibitory effect.

In this study, we aimed to evaluate the inhibitory effects of intracellular zinc concentration on EV-D68 replication. We also aimed to enhance the antiviral activity of zinc salts using a zinc ionophore. Our results collectively provide proofof-concept evidence for the clinical investigation of zinc supplementation in EV-D68 therapy.

\section{MATERIALS AND METHODS}

\section{Cells and Viruses}

RD cells (CCL-136, ATCC, Manassas, VA, United States), A549 cells (CCL-185, ATCC), and HeLa cells (CCL-2, ATCC) were cultured in Dulbecco's modified Eagle medium (DMEM; cat no: SH30022.01, HyClone, Logan, UT, United States) supplemented with $10 \%$ fetal bovine serum (FBS, REF: 04-001-1, Biological Industries, Beit HaEmek, Israel) and 1\% penicillin/ streptomycin solution. EV-D68 prototype Fermon (VR-1826, ATCC) and EV-D68 2014 US circulating strains, US/MO/1418947(VR-1823D, ATCC) and US/KY/14-18953(VR-1825D, ATCC), were amplified in RD cells. A mixture of cell and supernatant was collected approximately 3 days post-infection and subjected to multiple cycles of freezing and thawing. Then, the samples were clarified using low-speed centrifugation and passed through a $0.22-\mu \mathrm{m}$ filter, and viral particles were pelleted through a $20 \%$ sucrose cushion in an SW 28 rotor by centrifuging at $28,000 \mathrm{rpm}$ for $90 \mathrm{~min}$. The virus stocks were stored at $-80^{\circ} \mathrm{C}$ until further use. All the experiments in this study were approved by the ethics committee of the First Hospital of Jilin University.

\section{Cell Viability Assay}

The cells were treated with dose-gradient zinc when the confluency of RD cells was $80 \%$. We prepared quadruplicate wells for each dose in a 96-well plate and filled each well with $100 \mu \mathrm{l}$ medium. A blank group was prepared without cells but filled with medium. Before adding zinc solution, the wells were washed off once with phosphate-buffered saline (PBS) to prevent the interference of dead cells. After $24 \mathrm{~h}$ of incubation, the wells were washed off once with PBS and $10 \mu \mathrm{l}$ MTS (Biocompare, San Francisco, CA, United States) was added into each well, in dark conditions. Over the next $4 \mathrm{~h}$, the OD value was tested at $490 \mathrm{~nm}$ by a 550 Bio-Rad plate reader every hour. The data were analyzed with GraphPad Prism 8.0 software (GraphPad Software, San Diego, CA, United States), and the $\mathrm{IC}_{50}$ was calculated.

\section{Virus Titer Assay}

Viruses from the supernatants were isolated as previously described (Jiang et al., 2020). The supernatant was subjected to three freeze-thaw cycles. Then, the samples were centrifuged at $3,000 \mathrm{rpm}$ for $10 \mathrm{~min}$, and the supernatants were collected. Virus titers were determined based on the appearance of cytopathic effects (CPEs) in RD cells using a microtitration analysis in accordance with the Reed-Muench method (Reed and Muench, 1938). Virus titers were expressed as the $50 \%$ tissue culture infectious dose $\left(\mathrm{TCID}_{50}\right)$.

\section{Viral RNA Quantification}

Total RNA was extracted using a viral RNA isolation kit (Foregene, Chengdu, Sichuan, China) according to the manufacturer's protocol. cDNA was generated using a reverse transcriptase kit (TransGen, Beijing, China). The viral RNA was quantified using qRT-PCR with the SYBR Green reaction mix (GenStar, Beijing, China) in a Roche LightCycler 480 (Roche, Basel, Switzerland). The following primer was used: $G A P D H$ forward primer 5'-GCAAATTCCATGGCACCGT-3'; GAPDH reverse primer 5'-TCGCCCCACTTGATTTTGG-3'; EV-D68 forward primer 5'-TGTTCCCACGGTTGAAAACAA-3'; and EV-D68 reverse primer 5'-TGTCTAGCGTCTCATGGTTTTCAC-3'. The relative levels of EV-D68 RNA in different samples were determined using a comparative $2^{-\triangle \triangle \mathrm{CT}}$ method and normalized to the GAPDH gene (Wei et al., 2016).

\section{Immunoblotting}

Cells were harvested at various time points post-infection, washed twice with cold PBS, and lysed in lysis buffer [150 mM Tris ( $\mathrm{pH} 7.5$ ) containing $150 \mathrm{mM} \mathrm{NaCl}, 1 \%$ Triton X-100, and complete protease inhibitor cocktail (Roche)] and loading buffer [0.08 M Tris ( $\mathrm{pH} 6.8$ ) containing 2.0\% SDS, $10 \%$ glycerol, $0.1 \mathrm{M}$ DTT, and $0.2 \%$ bromophenol blue]. The solutions were boiled and vortexed for $10 \mathrm{~min}$ and then centrifuged at $12,000 \mathrm{rpm}$ for $10 \mathrm{~min}$. Supernatant proteins were separated using SDS-PAGE and transferred to nitrocellulose membranes using a semi-dry apparatus (Bio-Rad, Hercules, CA, United States). The membranes were probed with primary antibodies [anti-EV-D68 VP1 polyclonal antibody (GTX132312, Genetex, Irvine, CA, United States) and anti- $\alpha$-tubulin monoclonal antibody (A01410, GenScript, Nanjing, Jiangsu, China)] at $4^{\circ} \mathrm{C}$ overnight, followed by incubation with secondary antibodies (alkaline phosphataseconjugated goat anti-rabbit IgG (code: 115-005-045, Jackson ImmunoResearch Laboratories, West Grove, PA, United States) and goat anti-mouse IgG (code: 115-055-062, Jackson ImmunoResearch Laboratories, West Grove, PA, United States), respectively for $1 \mathrm{~h}$ at $25^{\circ} \mathrm{C}$. The membranes were stained with 5-bromo-4-chloro-3-indolyl phosphate and nitrotetrazolium blue chloride (Sigma-Aldrich, St. Louis, MO, United States) and visualized for band quantification. 


\section{Viral Attachment Assays}

For virus attachment experiments, cells were first rinsed with cold PBS and then incubated with viruses (multiplicity of infection, $\mathrm{MOI}=0.1$ ) at 4 or $37^{\circ} \mathrm{C}$ for $2 \mathrm{~h}$. Following this, the infected cells were washed with cold DMEM to remove unbound viruses. Total RNA was extracted using a viral RNA isolation kit (Foregene, Chengdu, Sichuan, China). The bound viral RNA was determined using qRT-PCR as described above.

\section{Statistical Analysis}

All statistical analyses were performed using GraphPad Prism software 8.0 (GraphPad Software, San Diego, CA, United States). Differences among test groups were analyzed using ANOVA. A value of $p<0.05$ was considered statistically significant.

\section{RESULTS}

\section{Zinc lons Inhibit Replication of EV-D68}

To investigate the effects of zinc salts on EV-D68 infectivity, $\mathrm{RD}$ cells were treated with zinc chloride $\left(\mathrm{ZnCl}_{2}\right)$, followed by challenge with an EV-D68 prototype Fermon $(\mathrm{MOI}=0.1)$. The CPEs were detected at $48 \mathrm{~h}$ post-infection (hpi) in the control group, whereas $\mathrm{ZnCl}_{2}$-treated cells showed prominent resistance to CPEs (Figure 1A). The titers of total viruses were significantly suppressed upon $\mathrm{ZnCl}_{2}$ treatment (Figure 1B). Consistently, the viral structural protein VP1 in the supernatant of infected cells was also reduced (Figure 1C). The $\mathrm{EC}_{50}$ value of $\mathrm{ZnCl}_{2}$ against EV-D68 replication was approximately $0.033 \mathrm{mM}$ (Figure 1D). We also measured $\mathrm{ZnCl}_{2}$ cytotoxicity using the MTS assay and determined that the $\mathrm{IC}_{50}$ of $\mathrm{ZnCl}_{2}$ was $0.26 \mathrm{mM}$ (Figure 1E), which was much higher than the $\mathrm{EC}_{50}$ of the antiviral activity. The anti-EV-D68 activity of $\mathrm{ZnCl}_{2}$ was also observed in other permissive cells, namely A549 cells and HeLa cells (Supplementary Figure S1). Furthermore, we also confirmed the inhibitory effect of zinc sulfate and zinc acetate on EV-D68 replication (Table 1). Collectively, zinc ions could potently inhibit EV-D68.

\section{Zinc lons Suppress EV-D68 Entry}

We sought to determine the phase of the EV-D68 life cycle that was inhibited upon $\mathrm{ZnCl}_{2}$ treatment. $\mathrm{ZnCl}_{2}$-pretreated $\mathrm{RD}$ cells were incubated with equal amounts of EV-D68 particles at 4 and $37^{\circ} \mathrm{C}$ (Figure 2A). Two hours later, the unbound virions were washed off with cold PBS, and the attachment and entry of the viral RNA were determined using qRT-PCR. The results indicated that both virus attachment and entry were inhibited by $\mathrm{ZnCl}_{2}$ treatment (Figures 2B,C), compared to the control groups.

To eliminate the potential effects of the interaction between zinc ions and EV-D68 particles on the antiviral activity of $\mathrm{ZnCl}_{2}$, we first incubated EV-D68 virions with $\mathrm{ZnCl}_{2}$ for $2 \mathrm{~h}$ before processing the virus attachment or entry assay (Figure 2D). Pre-incubation with $\mathrm{ZnCl}_{2}$ had no significant influence on EV-D68 entry (Figures 2E,F). Thus, our results suggested that zinc ions inhibited EV-D68 entry apparently due to the effect of $\mathrm{ZnCl}_{2}$ on the ability of cells to support virus replication rather than a direct effect on the virus itself.

\section{Zinc Ions Also Interfere with Release of EV-D68}

As our data showed that the inhibition of EV-D68 entry was milder than the suppression of EV-D68 replication by zinc ions, we suspected that zinc salts may also block viral replication at other steps in the life cycle of EV-D68. The levels of VP1 were used as indicators of the intracellular viral protein synthesis and viral particle release. RD cells were challenged with EV-D68 $(\mathrm{MOI}=0.1)$. After $48 \mathrm{~h}$, the cells and supernatant were harvested for the immunoblotting assay. $\mathrm{ZnCl}_{2}$ showed mildly effects on the expression of VP1 in the cell lysates, but potently decreased extracellular VP1 (Figures 3A,B). We then measured the changes in VP1 levels during infection in the presence or absence of $\mathrm{ZnCl}_{2}$ at different times after infection and found that $\mathrm{ZnCl}_{2}$ inhibited extracellular VP1 expression levels more potently than intracellular VP1. We also found that $\mathrm{ZnCl}_{2}$ exerted an inhibitory effect on the levels of extracellular VP1 after $8 \mathrm{~h}$, which was toward the end of the first life cycle of EV-D68 (Figures 3C-E). In summary, zinc ions seemed to exhibit dual antiviral activity against EV-D68 during the viral entry and release steps.

\section{Zinc lons Inhibit Isolate 2014 US Circulating Strains of EV-D68}

Recently, we and others demonstrated that the EV-D68 prototype virus and 2014 US circulating strains maintain different sensitivities for sialic acid-mediated viral entry (Wei et al., 2016). To evaluate the broad anti-EV-D68 activity of $\mathrm{ZnCl}_{2}$, we examined its antiviral activity against viral infection by primary EV-D68 isolates (US/MO/14-18947 [MO] and US/ $\mathrm{KY} / 14-18953[\mathrm{KY}])$. The results showed that $\mathrm{ZnCl}_{2}$ treatment protected RD cells against both 2014 US circulating strains of EV-D68, as demonstrated by decreases in related CPEs (Figure 4A). The viral protein VP1 was also reduced by zinc ions (Figures 4B,C). Further, we also observed significantly higher suppression of VP1 levels in the supernatant than in the cell lysates, which further supported our conclusion that zinc ions maintained dual antiviral activities on virus entry and release. Furthermore, $\mathrm{ZnCl}_{2}$ treatment significantly decreased the virus titers of total virions of the $\mathrm{MO}$ and $\mathrm{KY}$ strains (Figures 4D,E), compared to the control group.

\section{Pyrrolidine Dithiocarbamate Enhances the Antiviral Activity of Zinc lons Against EV- D68}

Pyrrolidine dithiocarbamate (PDTC), a zinc ionophore, has been shown to restrict infection by different human viruses by facilitating the import of zinc ions into cells (Lanke et al., 2007; Qiu et al., 2013). We investigated whether PDTC could strengthen the anti-EV-D68 activity of zinc ions. We used a much lower concentration of $\mathrm{ZnCl}_{2}(0.0005 \mathrm{mM})$ to treat $\mathrm{RD}$ cells, which showed no protective effect against EV-D68 infection (Figure 5). Meanwhile, a single treatment 
A

A $\mathrm{ZnCl}_{2}$
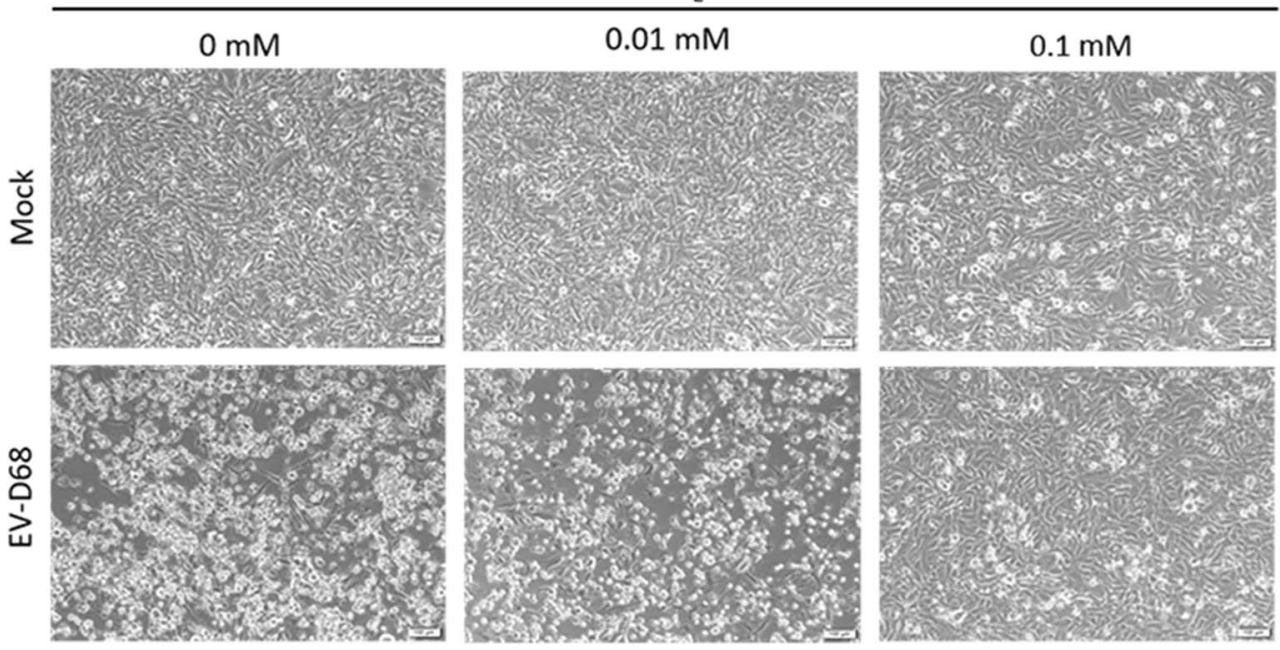

B
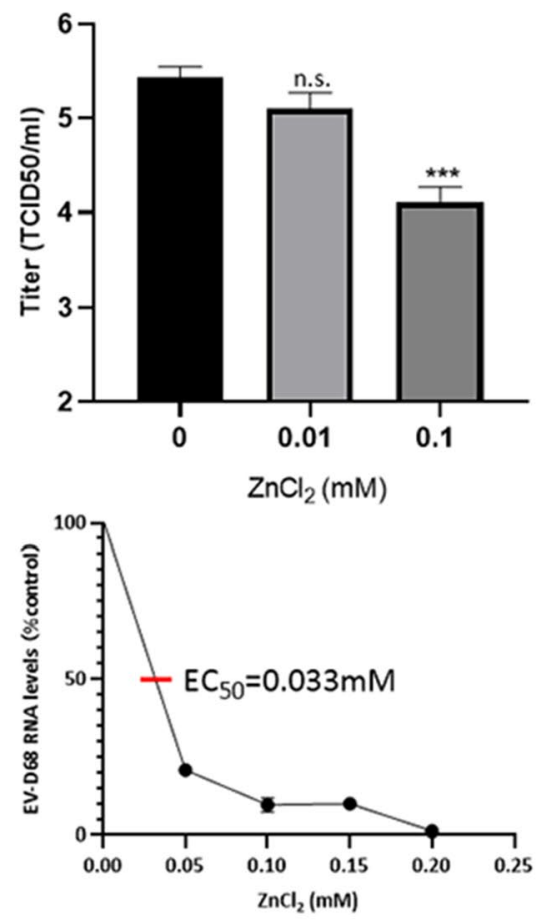

C

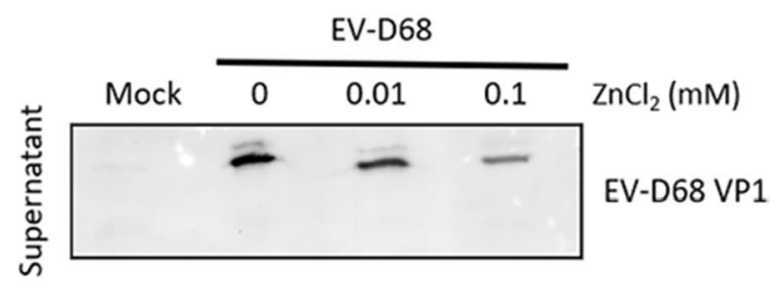

E

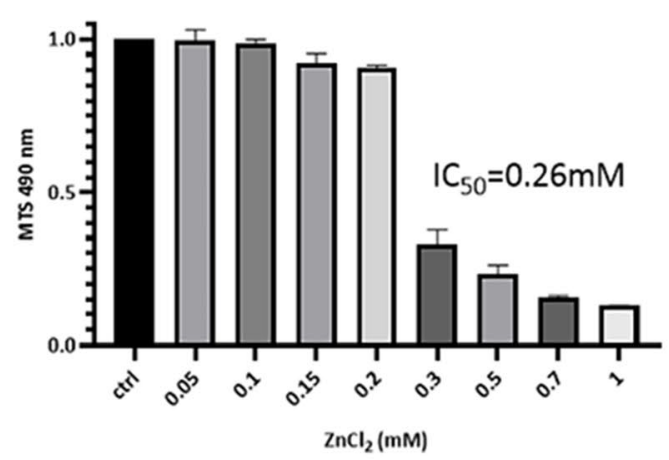

FIGURE 1 | $\mathrm{ZnCl}_{2}$ inhibits enterovirus D68 (EV-D68) replication. (A) After a 2-h exposure to EV-D68, RD cells were treated with ZnCl 2 . The cells were incubated under standard conditions. Cytopathic effects (CPEs) were observed $48 \mathrm{~h}$ post-infection (hpi). (B) Determination of progeny viral production. Supernatants were collected $48 \mathrm{hpi}$, and viral titers were determined by standard plaque assay. Error bars indicate the $\operatorname{SD}\left({ }^{\star} p<0.05,{ }^{* \star} p<0.01,{ }^{* \star *} p<0.001\right.$ and $\left.{ }^{* \star * \star} p<0.0001\right)$.

(C) VP1 expression in the supernatant of $\mathrm{ZnCl}_{2}$-treated RD cells. The supernatant was collected at $48 \mathrm{hpi}$, and the samples were prepared for western blot.

(D) Effect of zinc on EV-D68 RNA replication $\left(\mathrm{EC}_{50}=0.033 \mathrm{mM}\right)$. (E) Cell viability assay. Cellular toxicity of zinc chloride was evaluated by MTS assay (IC50=0.26mM).

with PDTC at a concentration of $65 \mu \mathrm{M}$ also showed no influence on viral infection. Intracellular VP1 expression was suppressed only in the presence of both PDTC and $\mathrm{ZnCl}_{2}$, compared to the control group (Figure 5A). PDTC and $\mathrm{ZnCl}_{2}$ treatment significantly enhanced the resistance of $\mathrm{RD}$ cells to EV-D68 infection (Figure 5B). We noted the fold change of the titers of released virion is higher than that of intracellular VP1 expression in presence of PDTC treatment, which further supports our conclusion that EV-D68 inhibition by zinc influx is mainly targeting at virus release step. In addition, we confirmed that the zinc ions from FBS were associated with the antiviral activity of PDTC (Figure 5C). Hence, enhanced zinc ions influx could boost host cells against EV-D68 infection. 
TABLE 1 | Effect of various zinc salts on EV-D68 replication.

\begin{tabular}{|c|c|c|}
\hline \multirow{2}{*}{ Salt } & Concentration & Virus titer \\
\hline & $(\mathrm{mM})$ & (48hpi.) \\
\hline \multirow[t]{3}{*}{ Chloride } & 0 & 5.5 \\
\hline & 0.01 & 5 \\
\hline & 0.1 & 4 \\
\hline \multirow[t]{3}{*}{ Sulfate } & 0 & 5 \\
\hline & 0.01 & 4.5 \\
\hline & 0.1 & 4 \\
\hline \multirow[t]{3}{*}{ Acetate } & 0 & 5 \\
\hline & 0.01 & 4.3 \\
\hline & 0.1 & 4 \\
\hline
\end{tabular}

Zinc salts exert inhibitory effect on EV-D68 replication. The EV-D68-infected RD cells were treated with various zinc salts (zinc chloride, zinc sulfate, and zinc acetate). At $48 \mathrm{hpi}$, the supernatant was collected for virus titer assay.

\section{DISCUSSION}

Enterovirus D68 and EV-A71 are the two major non-polio enteroviruses that circulate periodically around the world. Due to the current coronavirus disease of 2019 (COVID-19) pandemic, the number of reported EV-D68 cases has decreased in recent years. However, EV-D68 is still a non-negligible threat to public health due to its rapid spread and irreversible neurological toxicity. In our study, we demonstrated that zinc ions can suppress CPE and infectious virus generation by EV-D68 in vitro. Different zinc salts, namely zinc chloride, zinc sulfate, and zinc acetate, had identical effects on EV-D68 inhibition (Table 1). The inhibition was concentration-dependent and broad-spectrum for both the prototype and 2014 US circulating EV-D68 strains (Figure 4). Hence, our results support the
A

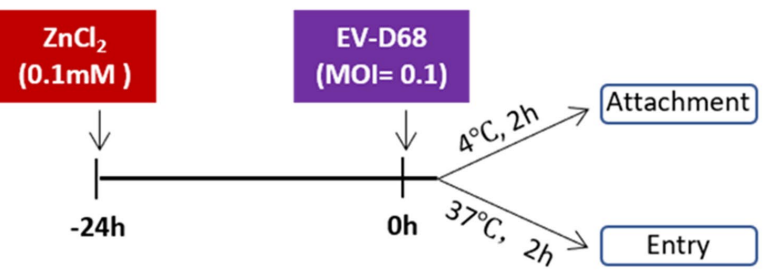

B

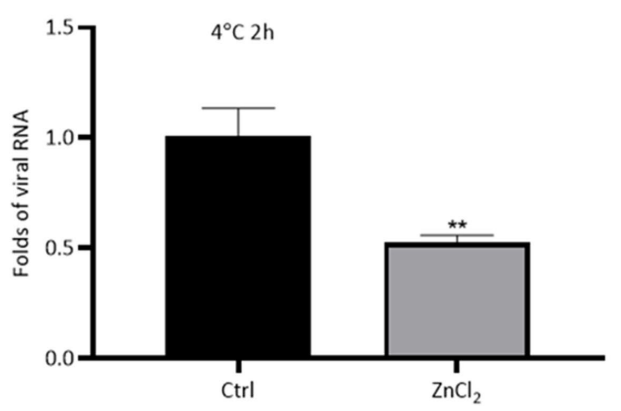

C

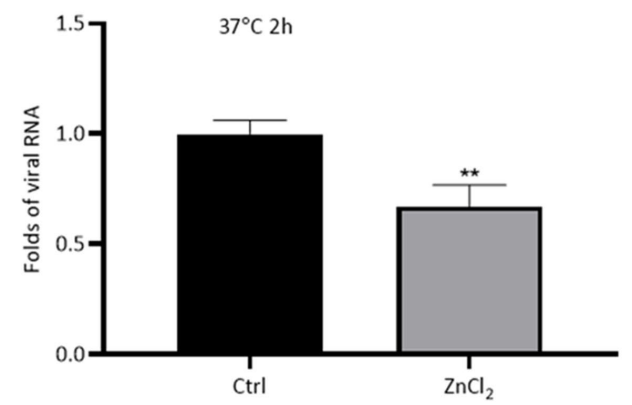

D

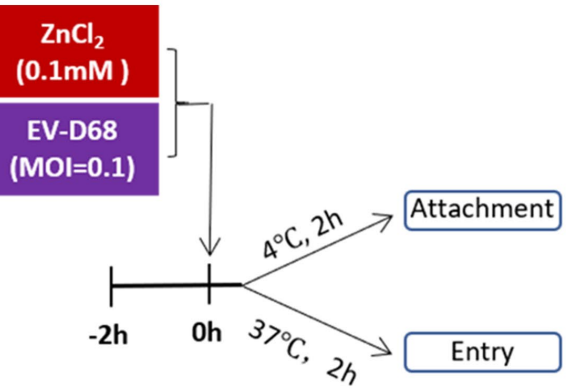

$\mathbf{E}$

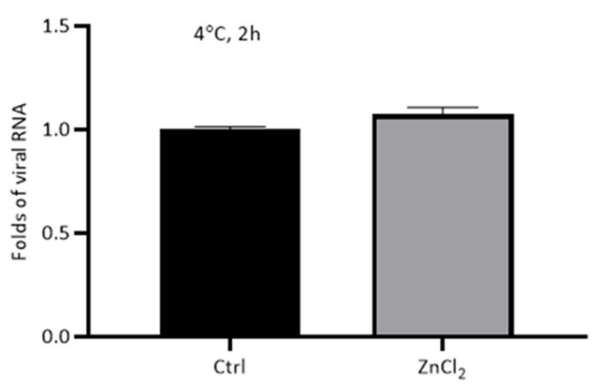

$\mathbf{F}$

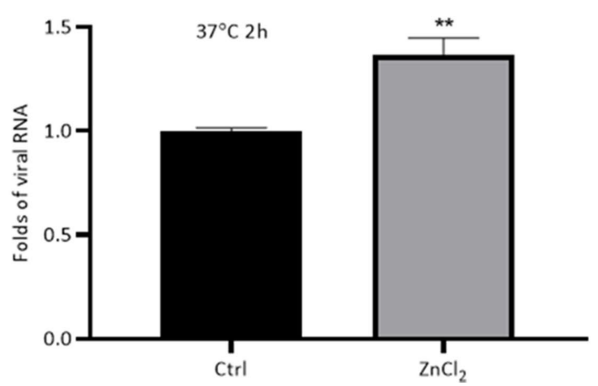

FIGURE 2 | Zinc ions suppress EV-D68 entry. (A-C) RD cells pretreated with $\mathrm{ZnCl}_{2}$ for $24 \mathrm{~h}$. RD cells were precooled at $4^{\circ} \mathrm{C}$ for $30 \mathrm{~min}$ before challenging with Fermon strain of EV-D68, and the cells were kept at 4 or $37^{\circ} \mathrm{C}$ for $2 \mathrm{~h}$. Unattached virus particles were washed off. The cells were collected for qRTPCR assay to detect the viral replication. (D-F) EV-D68 was incubated with $\mathrm{ZnCl}_{2}$ for $2 \mathrm{~h}$ at $37^{\circ} \mathrm{C}$. The precooled RD cells were treated with this mixture at 4 or $37^{\circ} \mathrm{C}$ for $2 \mathrm{~h}$. The cells were collected at $2 \mathrm{hpi}$ for qRT-PCR assay. Error bars indicate the standard deviation $\left({ }^{*} p<0.05 ;{ }^{* \star} p<0.01\right.$; ${ }^{\star \star \star} p<0.001$ and $\left.{ }^{\star \star \star *} p<0.0001\right)$. 
A
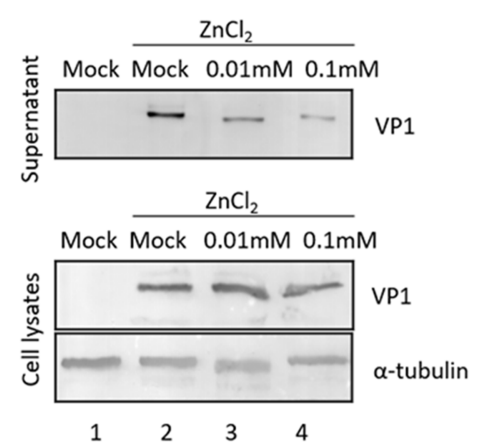

B

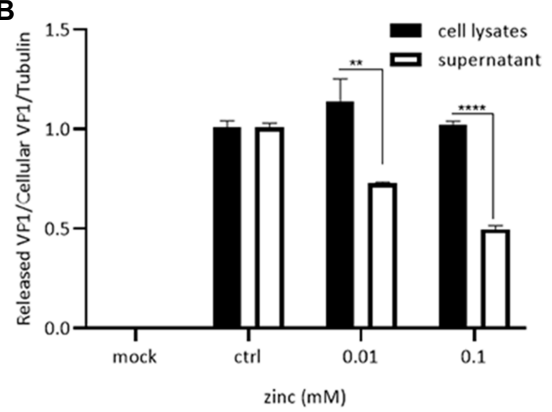

C

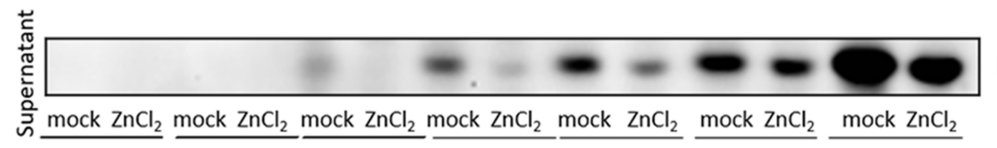

EV-D68 VP1
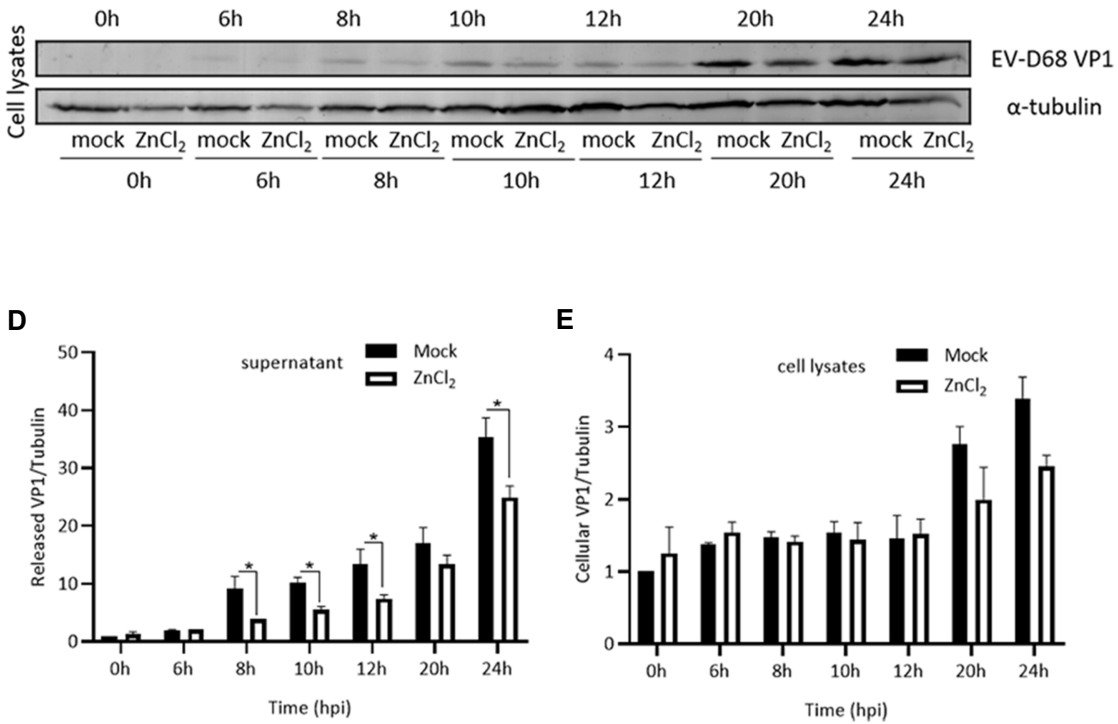

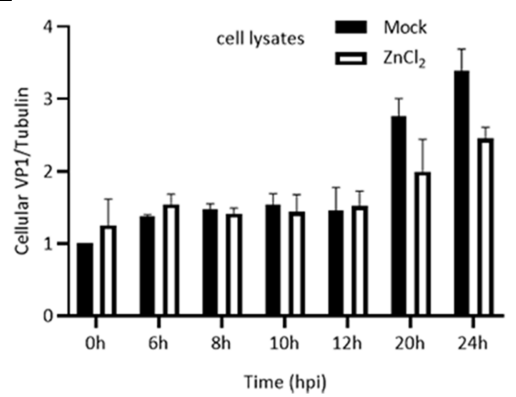

FIGURE 3 | Zinc ions suppress release of EV-D68. (A) VP1 expression in supernatant and cell lysate at $48 \mathrm{hpi}$ (B) The level of viral capsid protein expression from supernatant and cell lysate under same $\mathrm{ZnCl}_{2}$-treated concentration was compared with each other. (C) The changes of VP1 expression of the supernatant and cell lysates between the $\mathrm{ZnCl}_{2}$ treatment group and the control group at different times after infection. $\mathrm{RD}$ cells were treated with $\mathrm{ZnCl}_{2}$ at several specific time points, and the viral capsid protein expression level of supernatant (D) and cell lysates (E) was compared with that of the control group by Image $\mathrm{J}$.

notion that zinc supplementation could serve as a potential prophylactic and therapeutic treatment against EV-D68 infection.

Reportedly, zinc supplementation inhibits the replication of diverse viruses, such as herpes simplex virus (HSV), human immunodeficiency virus (HIV), and vaccinia virus (Korant et al., 1974; Katz and Margalith, 1981; Kumel et al., 1990; Baum et al., 2010). Zinc supplementation interferes with these viruses at different stages, including inhibition of viral uncoating, viral genome transcription, viral protein translation, and viral polyprotein processing (Fridlender et al., 1978; Kumel et al., 1990; Arens and Travis, 2000; Yuasa et al., 2006; Lanke et al., 2007; Liu and Kielian, 2012). Here, we found that zinc ions decreased the capacity of EV-D68 to attach to the targeted cells, which is the initial step for EV-D68 entry (Figure 2). Similar to this behavior, previous studies revealed that zinc ions can fit into the canyons on the surface of rhinovirus virions, thereby preventing the binding of viruses to the cell surface (Kumel et al., 1990). These results were consistent with previous cryo-electron microscopy structural studies that showed high similarity between the surface conformation of EV-D68 and rhinovirus (Liu et al., 2015b). Furthermore, we confirmed that the inhibition relied on the effect of $\mathrm{ZnCl}_{2}$ on the ability of cells to support virus replication rather than a direct effect on the virus itself.

In addition to the inhibition of EV-D68 entry, we also found that zinc treatment suppressed the total virion release from producing cells, by comparing the expression rates of VP1 in the cells and supernatant during viral replication (Figure 3). Hence, zinc ions potently blocked EV-D68 infection through the dual inhibition of virus entry and release. The detailed intracellular factors targeted by zinc ions, which are essential for its antiEV-D68 activity, warrant further investigation. Nevertheless, the present study further enriched our understanding of the mechanisms of action of zinc salts against viral infections.

The physiological level of zinc in plasma is $10-20 \mu \mathrm{M}$ (Sugarman, 1983), and only about $10-20 \%$ of ingested zinc is absorbed. Increasing the sensitivity of host cells to EV-D68 inhibition by zinc salts could be helpful for the application of zinc salts in antiviral treatment. We found that the zinc ionophore PDTC, which facilitates the influx of zinc ions into cells, strongly amplified the antiviral activity of zinc. The concentration of $\mathrm{ZnCl}_{2}$ we used was much lower than the $\mathrm{EC}_{50}$, showing no significant effect on EV-D68 infection in single-treated cells, but potently suppressing virus infection in the presence of PDTC. These results showed that increasing the zinc influx may be a potential effective strategy for antiviral treatment.

It is important to further investigate EV-D68 infection and clinical outcomes in populations with zinc deficiency and in infants with zinc supplementation. Considering the safety of zinc usage, its easy availability, and the antiviral activity, the work described herein provides compelling evidence that zinc supplementation is an optimal therapeutic candidate for treating EV-D68 infections. 
A

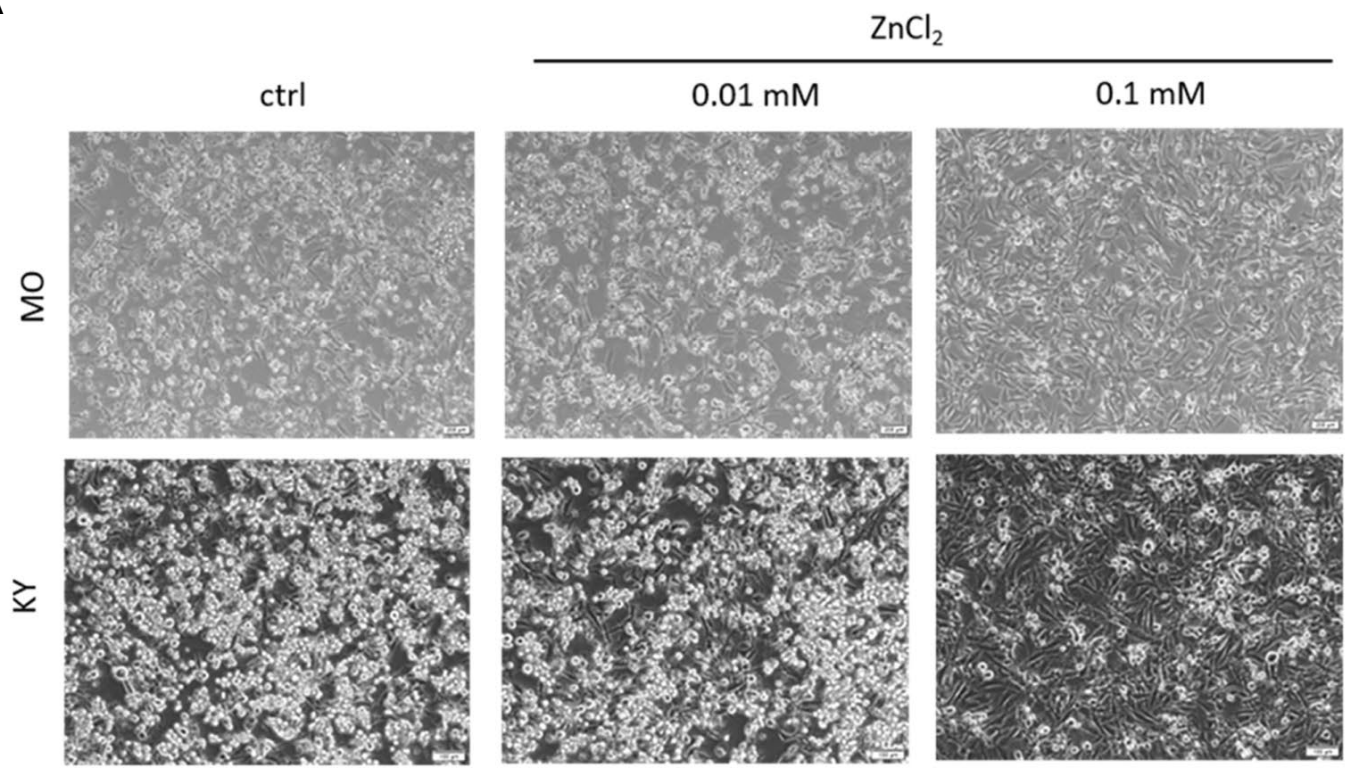

B

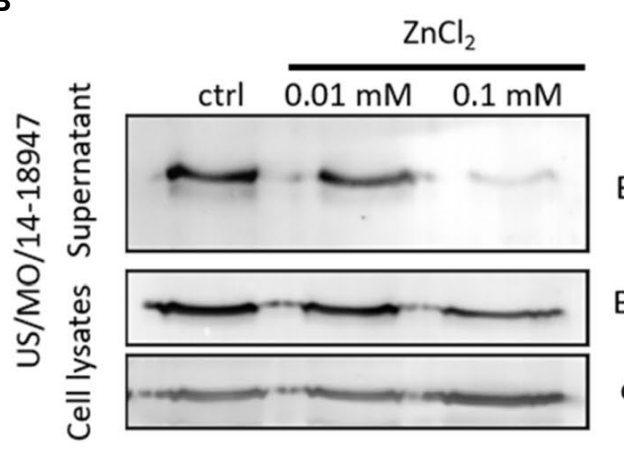

D

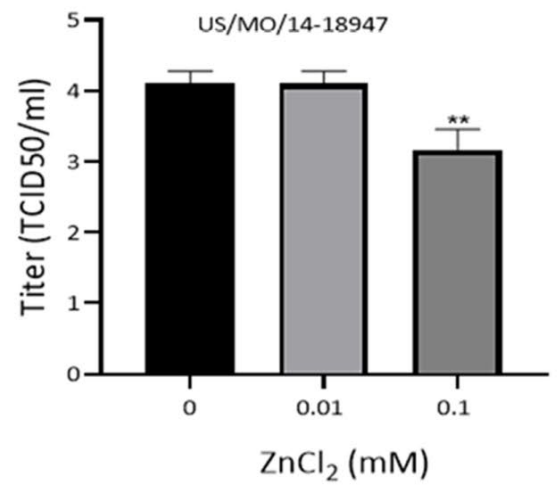

C

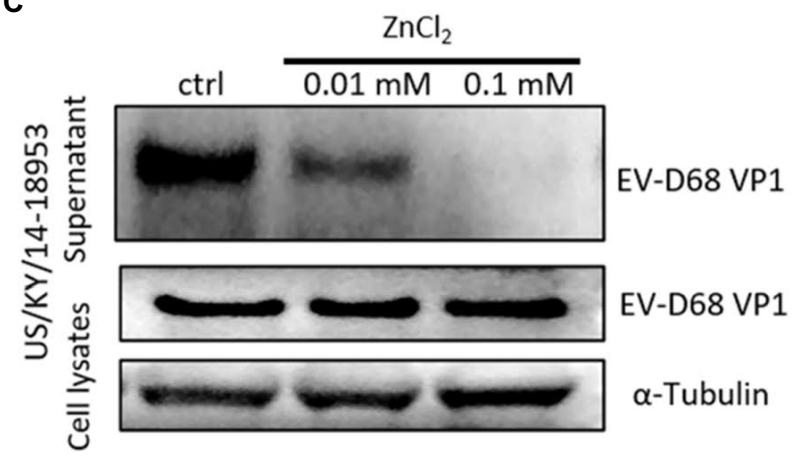

$\mathbf{E}$

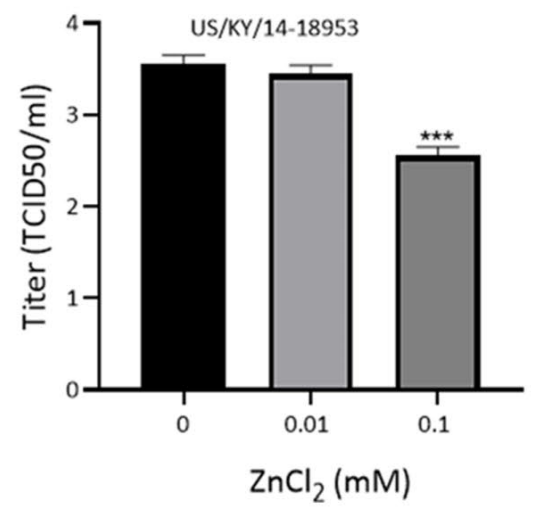

FIGURE 4 | Zinc exerts inhibitory effects on US/MO/14-18947 and US/KY/14-18953 strains. (A) Cytopathic effects were observed at 48 hpi. (B,C) Immunoblotting showing VP1 expression at $48 \mathrm{hpi}$. (D,E) Determination of progeny viral production. Supernatants were collected at $48 \mathrm{hpi}$, and viral titers were determined by standard plaque assay. Error bars indicate the SD $\left({ }^{*} p<0.05 ;{ }^{* \star} p<0.01 ;{ }^{* \star} p<0.001\right.$ and $\left.{ }^{* \star \star *} p<0.0001\right)$.

\section{DATA AVAILABILITY STATEMENT}

The original contributions presented in the study are included in the article/Supplementary Material; further inquiries can be directed to the corresponding authors.

\section{AUTHOR CONTRIBUTIONS}

WW, SL, and HG performed manuscript writing. WW, SL, $\mathrm{HG}$, and XC carried out data analysis. WW contributed to study design. SL and HG performed data collection. All 


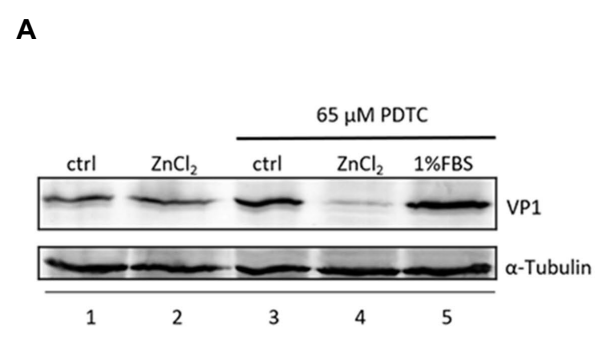

B

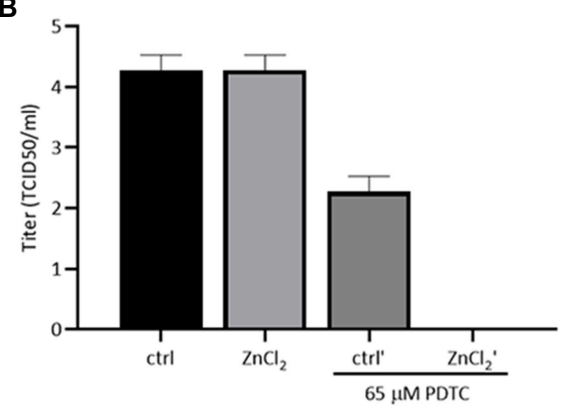

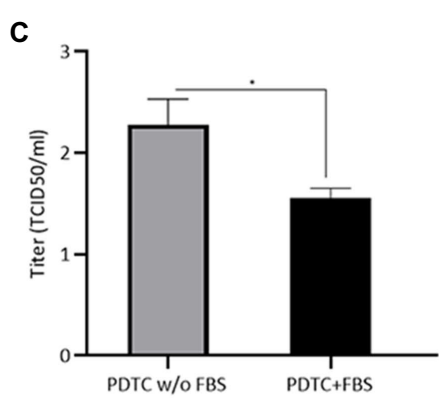

FIGURE 5 | Combined treatment of zinc and pyrrolidine dithiocarbamate (PDTC) inhibits EV-D68 replication. (A) The combination of PDTC and zinc has an inhibitory effect on cellular VP1 expression. (B) The combination of PDTC and zinc decreases the amount of released virion of infected RD cells. (C) Infected cells were treated with PDTC with or without fetal bovine serum (FBS). The zinc ion in FBS helps PDTC to exert viral inhibitory effect. Error bars indicate the SD $\left({ }^{\star} p<0.05 ;{ }^{* \star} p<0.01 ;{ }^{* \star *} p<0.001\right.$ and $\left.{ }^{\star \star \star *} p<0.0001\right)$.

authors contributed to the article and approved the submitted version.

\section{FUNDING}

This work was supported in part by funding from the National Natural Science Foundation of China (81772183 and 31800150), the National Science and Technology Major Project (2018ZX10731-101-001-016), the open project of Key Laboratory of Organ Regeneration and Transplantation, Ministry of Education (20202005), and the Department of Science and

\section{REFERENCES}

Arens, M., and Travis, S. (2000). Zinc salts inactivate clinical isolates of herpes simplex virus in vitro. J. Clin. Microbiol. 38, 1758-1762. doi: 10.1128/ JCM.38.5.1758-1762.2000

Baggen, J., Thibaut, H. J., Staring, J., Jae, L. T., Liu, Y., Guo, H., et al. (2016). Enterovirus D68 receptor requirements unveiled by haploid genetics. Proc. Natl. Acad. Sci. U. S. A. 113, 1399-1404. doi: 10.1073/pnas. 1524498113

Baum, M. K., Lai, S., Sales, S., Page, J. B., and Campa, A. (2010). Randomized, controlled clinical trial of zinc supplementation to prevent immunological failure in HIV-infected adults. Clin. Infect. Dis. 50, 1653-1660. doi: 10.1086/652864

Bracha, M., and Schlesinger, M. J. (1976). Inhibition of Sindbis virus replication by zinc ions. Virology 72, 272-277. doi: 10.1016/0042-6822(76)90330-5

Chang, K. H., Lu, F. J. H., Chyi, T., Hsu, Y. W., Chan, S. W., and Wang, E. T. W. (2017). Examining the stress-burnout relationship: the mediating role of negative thoughts. PeerJ 5:e4181. doi: 10.7717/peerj.4181

D'Esposito, M., and Postle, B. R. (2015). The cognitive neuroscience of working memory. Annu. Rev. Psychol. 66, 115-142. doi: 10.1146/annurevpsych-010814-015031

Fenstermacher, K. J., and DeStefano, J. J. (2011). Mechanism of HIV reverse transcriptase inhibition by zinc: formation of a highly stable enzyme-(primertemplate) complex with profoundly diminished catalytic activity. J. Biol. Chem. 286, 40433-40442. doi: 10.1074/jbc.M111.289850

Fridlender, B., Chejanovsky, N., and Becker, Y. (1978). Selective inhibition of herpes simplex virus type 1 DNA polymerase by zinc ions. Virology 84, 551-554. doi: 10.1016/0042-6822(78)90274-X

Guo, H., Li, Y., Liu, G., Jiang, Y., Shen, S., Bi, R., et al. (2019). A second open reading frame in human enterovirus determines viral replication in
Technology of Jilin Province (Nos. 20190304033YY and 20180101127JC).

\section{ACKNOWLEDGMENTS}

We thank Y. Jiang, Y. Li, and G. Liu for technical assistance.

\section{SUPPLEMENTARY MATERIAL}

The Supplementary Material for this article can be found online at: https://www.frontiersin.org/articles/10.3389/fmicb.2021.748546/ full\#supplementary-material intestinal epithelial cells. Nat. Commun. 10:4066. doi: 10.1038/ s41467-019-12040-9

Haraguchi, Y., Sakurai, H., Hussain, S., Anner, B. M., and Hoshino, H. (1999). Inhibition of HIV-1 infection by zinc group metal compounds. Antivir. Res. 43, 123-133. doi: 10.1016/S0166-3542(99)00040-6

Jiang, Y., Liu, S., Shen, S., Guo, H., Huang, H., and Wei, W. (2020). Methylbeta-cyclodextrin inhibits EV-D68 virus entry by perturbing the accumulation of virus particles and ICAM-5 in lipid rafts. Antivir. Res. 176:104752. doi: 10.1016/j.antiviral.2020.104752

Katz, E., and Margalith, E. (1981). Inhibition of vaccinia virus maturation by zinc chloride. Antimicrob. Agents Chemother. 19, 213-217. doi: 10.1128/ AAC.19.2.213

Korant, B. D., Kauer, J. C., and Butterworth, B. E. (1974). Zinc ions inhibit replication of rhinoviruses. Nature 248, 588-590. doi: 10.1038/248588a0

Kumel, G., Schrader, S., Zentgraf, H., Daus, H., and Brendel, M. (1990). The mechanism of the antiherpetic activity of zinc sulphate. J. Gen. Virol. 71, 2989-2997. doi: 10.1099/0022-1317-71-12-2989

Lanke, K., Krenn, B. M., Melchers, W. J. G., Seipelt, J., and van Kuppeveld, F. J. M. (2007). PDTC inhibits picornavirus polyprotein processing and RNA replication by transporting zinc ions into cells. J. Gen. Virol. 88, 1206-1217. doi: 10.1099/vir.0.82634-0

Liu, C. Y., and Kielian, M. (2012). Identification of a specific region in the el fusion protein involved in zinc inhibition of semliki forest virus fusion. J. Virol. 86, 3588-3594. doi: 10.1128/JVI.07115-11

Liu, Y., Sheng, J., Baggen, J., Meng, G., Xiao, C., Thibaut, H. J., et al. (2015a). Sialic acid-dependent cell entry of human enterovirus D68. Nat. Commun. 6:8865. doi: $10.1038 /$ ncomms 9865

Liu, Y., Sheng, J., Fokine, A., Meng, G., Shin, W. H., Long, F., et al. (2015b). Structure and inhibition of EV-D68, a virus that causes respiratory illness in children. Science 347, 71-74. doi: 10.1126/science.1261962 
Lulla, V., Dinan, A. M., Hosmillo, M., Chaudhry, Y., Sherry, L., Irigoyen, N., et al. (2019). An upstream protein-coding region in enteroviruses modulates virus infection in gut epithelial cells. Nat. Microbiol. 4, 280-292. doi: 10.1038/ s41564-018-0297-1

Midgley, S. E., Christiansen, C. B., Poulsen, M. W., Hansen, C. H., and Fischer, T. K. (2015). Emergence of enterovirus D68 in Denmark, June 2014 to February 2015. Euro. Surveill. 20:21105. doi: 10.2807/1560-7917.es2015.20.17.21105

Midgley, C. M., Jackson, M. A., Selvarangan, R., Turabelidze, G., Obringer, E., Johnson, D., et al. (2014). Severe respiratory illness associated with enterovirus D68-Missouri and Illinois, 2014. MMWR Morb. Mortal. Wkly Rep. 63, 798-799. doi: 10.1016/j.annemergmed.2014.12.007

Nafisa, S., Paul, P., and Sovani, M. (2021). A case report of acute flaccid paralysis caused by enterovirus D68 infection: the beginning of a polio-like epidemic? Cureus 13:e15625. doi: 10.7759/cureus.15625

Qiu, M., Chen, Y., Cheng, L., Chu, Y., Song, H. Y., and Wu, Z. W. (2013). Pyrrolidine dithiocarbamate inhibits herpes simplex virus 1 and 2 replication, and its activity may be mediated through dysregulation of the ubiquitinproteasome system. J. Virol. 87, 8675-8686. doi: 10.1128/JVI.00869-13

Reed, L. J., and Muench, H. (1938). A simple method of estimating fifty per cent endpoints. Am. J. Epidemiol. 27, 493-497. doi: 10.1093/oxfordjournals. aje.a118408

Schieble, J. H., Fox, V. L., and Lennette, E. H. (1967). A probable new human picornavirus associated with respiratory diseases. Am. J. Epidemiol. 85, 297-310. doi: 10.1093/oxfordjournals.aje.a120693

Suara, R. O., and Crowe, J. E. Jr. (2004). Effect of zinc salts on respiratory syncytial virus replication. Antimicrob. Agents Chemother. 48, 783-790. doi: 10.1128/aac.48.3.783-790.2004
Sugarman, B. (1983). Zinc and infection. Rev. Infect. Dis. 5, 137-147. doi: 10.1093/clinids/5.1.137

Wei, W., Guo, H., Chang, J., Yu, Y., Liu, G., Zhang, N., et al. (2016). ICAM-5/ Telencephalin is a functional entry receptor for enterovirus D68. Cell Host Microbe 20, 631-641. doi: 10.1016/j.chom.2016.09.013

Yuasa, K., Naganuma, A., Sato, K., Ikeda, M., Kato, N., Takagi, H., et al. (2006). Zinc is a negative regulator of hepatitis C virus RNA replication. Liver Int. 26, 1111-1118. doi: 10.1111/j.1478-3231. 2006.01352.x

Conflict of Interest: The authors declare that the research was conducted in the absence of any commercial or financial relationships that could be construed as a potential conflict of interest.

Publisher's Note: All claims expressed in this article are solely those of the authors and do not necessarily represent those of their affiliated organizations, or those of the publisher, the editors and the reviewers. Any product that may be evaluated in this article, or claim that may be made by its manufacturer, is not guaranteed or endorsed by the publisher.

Copyright (C) $2021 \mathrm{Liu}, \mathrm{Cao}, \mathrm{Guo}$ and Wei. This is an open-access article distributed under the terms of the Creative Commons Attribution License (CC BY). The use, distribution or reproduction in other forums is permitted, provided the original author(s) and the copyright owner(s) are credited and that the original publication in this journal is cited, in accordance with accepted academic practice. No use, distribution or reproduction is permitted which does not comply with these terms. 